\title{
Improving the Efficiency of Block Matching Algorithms using Optimization Techniques
}

\author{
Syed Ali Zia \\ Department of Computer Systems Engineering \\ University of Engineering \& Technology Peshawar,
}

Pakistan

\author{
Nasir Ahmad \\ Department of Computer Systems Engineering \\ University of Engineering \& Technology Peshawar,
}

Pakistan

\begin{abstract}
Block matching techniques are efficient methods among existing motion estimation methods according to literature. Block matching techniques mostly focuses on the factors like estimation accuracy and computational complexity. There are few methods among the existing search methods which can provide satisfactory result at a very low computational cost but none of these methods can effectively jump out of the local optimum when processing large motion sequences. The proposed search method mainly adopts threshold values for early termination of block-matching in order to remove unnecessary search and is evaluated and compared together in terms of signal to noise ratio and search point's avoidance per macro block for different block size and search area. Experimental results showed that the proposed method has achieved significant improvement on both Estimation accuracy and computational complexity.
\end{abstract}

\section{Keywords}

Motion Estimation, Block matching, fast search, Block Termination, Local Minima.

\section{INTRODUCTION}

Real Time application development has become a recent trend with the advent of technology. The vast array including such applications depends on broadcast of video sequences and images [1]. The need of technology in daily life practice can be credited for this advancement [2]. Therefore motion in video sequences plays important role in many artificial vision systems and that includes applications like surveillance, object tracking and reconstruction, robot navigation and video coding. Block matching technique offers many unconventional methods for detection of objects. The main process is to match the blocks of current frame to the blocks of the frame in reference and the aim behind this process is to find out the best matching block. In [3], block matching algorithm (BMA) preprocessing of the frame is done to reduce noise makes detection difficult [4]. All the blocks in current and reference frames are matched using some matching criteria in order to find out the best match [1]. There are main criteria for matching the blocks of current and reference frame. These criteria help to find out the best matching block from the current frame [3][5][11]. While this matching process, the blocks are moved from their location if the best suitable matched block is located on different position. For moving the best matched blocks to new location, a cover of movable blocks is formed. All the movable blocks are combined in a form of moving object so that it can be moved collectively from one location to another location.

\section{LITERATURE REVIEW}

Various leveled block matching built up the progressive hunt dependent on the accompanying two perceptions[6][7]. From one perspective, for a moderately vast dislodging, exact square coordinating requires a generally substantial square size[8]. This is possible in the event that one thinks about its contrary case: a substantial removal with a little relationship window. Under this situation, search is expensive [9][10][12]. Hence the likelihood of finding various matches is high, bringing about untrustworthy movement estimation. Then again, a substantial square size may disregard the supposition that all pixels in the square offer a similar dislodging vector [13]. Consequently a moderately little square is required for matching the suspicion. Following perceptions shed light on the issue of utilizing a settled square, which may prompt problematic movement estimation [14]. To fulfill these two negating prerequisites at the same time. For encouraging focus, consider multi leveled progressive square coordinating calculation, in which three square coordinating strategies are led, each with its own parameters. Square coordinating is first directed as for the biggest size of squares and connection windows. This pursuit strategy is done comparably, in view of the consequences of the second hunt[15].Block matching utilizes more time every now and again than some other movement estimation strategy in movement remunerated coding by parceling an edge into non-covered, similarly separated, settled size, little rectangular squares and accepting that every one of the pixels in a square ordeal the equivalent translational movement, square coordinating dodges the trouble experienced in movement estimation of selfassertively formed squares. Therefore, block matching is easier and less complex and includes less side data contrasted and movement estimation with subjectively molded squares. In spite of the fact that this basic model considers interpretation movement just, different kinds of movements, for example, turn and zooming of vast items, might be nearly approximated by the piecewise interpretation of these little squares, gave that these squares are little enough [15]. This critical perception, initially made by Jain and Jain, has been affirmed over and over from that point forward. For progressively precise movement estimation, the extent of $8 \mathrm{x}$ 8 is utilized now and again. In the last case, increasingly precise movement is acquired at the expense causing data and higher computational multifaceted nature. Few unique kinds of coordinating standards are utilized in square coordinating. As already mentioned and demonstrated that distinctive standards don't cause critical contrasts for coordination of blocks, the mean total distinction is henceforth favored because of its effortlessness in usage. From one viewpoint, a full-seek methodology conveys great exactness in hunting down the best match. Then again, it requires a lot of calculation. So as to bring down computational multifaceted nature, a few quick seeking methods were produced.

Other than these suboptimum look strategies, there are some different estimates created to bring down calculation by the method of sub-sampling in the first squares and the close gaps 
[16]. The method of sub-sampling helps to decrease the computational weight in square coordinating, while the precision of the assessed movement vectors might be influenced.

Subsequently, the sub-sampling methodology is suggested in those cases where number is large so the large samples can be broken down in small samples. In multi-resolution, a groundbreaking design loans itself well for quick inquiry in square coordinating. It fundamentally diminishes the calculations included.

Thresholding multi-resolution square coordinating further spares calculation. For accurate matching of the blocks decisions are based on one pixel and half pixel accuracy. Half and full pixel accuracy is achieved by the information of the location where the blocks are resided [5][16]. As per literature, more sub-sampling proves to give better and accurate results as number of calculations increase with the division in number of pixels.

There are few procedures which ensure success over the ordinary techniques and it is possible only with the increase in number of calculations for the pixels and blocks. Multiple methods are adopted for making calculations on number of pixels involved in any search pattern. In multi-step searching methods different block sizes are set to in order to check which one is best suitable in context of accuracy. The smaller window sizes and smaller ranges of estimation ensure accuracy.

The multi-grid square coordinating system utilizes multi-grid structure, another incredible computational structure in picture preparing for block coordination. In addition to the fact that it is ideal in the feeling of bit sparing, it wipes out the requirement for setting a limit. Clearly the square based model experiences a progressively serious issue along moving limits. To take care of the issue, the prescient movement field division procedure make the squares including moving limits have the movement field estimated with pixel goals rather than square goals. So as to spare shape overhead, division is done in reverse, i.e., in view of recently decoded edges. So as to maintain a strategic distance from an extensive increment of side data related with additional movement vectors.

Therefore, the strategy is equipped for reproducing discontinuities in the movement field at pixel goals while keeping up indistinguishable measure of movement vectors from the traditional square coordinating method. The last enhancement over customary square coordinating talked about in this part is covered square coordinating. As opposed to managing squares autonomously of one another, the covered square coordinating strategy augments squares in order to make them cover. A window work is then developed and utilized in both movement estimation and movement pay. Since it loosens up the limitation of a non-covered square parcel forced by regular square coordinating, it accomplishes preferred execution over the ordinary square coordinating.

Amid the most recent two decades, many quick calculations and VLSI designs have been proposed. In this paper, we attempt to give a broad investigation of movement estimation with our new advancements [18]. The primary ideas of quick calculations can be grouped into six classes: decrease in hunt positions, rearrangements of coordinating model, bit-width decrease, prescient pursuit, various leveled inquiry, and quick full pursuit. The systolic exhibits can be partitioned into between sort and intra-type with 1-D [11], 2-D [17], and tree structures [18]. Hexagonal plots are displayed for framework creators to obviously assess the designs in six viewpoints including entryway tally, required recurrence, equipment use, memory transmission capacity, memory bit-width, and dormancy. Next, models supporting quick calculations are additionally checked on.

Different leveled Search It is extraordinary that a multi objectives structure, generally called a pyramid structure, is a mind boggling computational course of action for picture taking care of errand [16][33]. To save the estimation of FSBMA, more often than not to swing to the pyramid structure. The multi objectives plot relies upon foreseeing a fundamental examination at the coarse measurement and refining the check at the fine measurement[18][19]. By and large a couple of measurement different leveled look is grasped. Honestly, the multi objectives framework has been seen as a champion among the most capable procedures in BMA and is generally gotten in applications with sweeping edges and interest zones[19][20]. In fast Full Search the primary thought of quick full inquiry calculations is expressed as pursues. In the beginning time, a straightforward check is done to $300[21][22][23]$ distinguish whether a hopeful square is conceivable to be the ideal one. At that point, just the potential applicant squares are additionally handled for nitty gritty bending count. Accordingly, a vast bit of pointless calculation for outlandish competitor squares can be dodged. Additionally, so as to diminish the span of hash table and along these lines accomplish accelerate, MSEA is connected with standardized halfway contortion. Truth be told, the consequences of quick full hunt are not actually equivalent to FSBMA. In some cases, minor contrasts happen [24].

\section{PROPOSED TECHNIQUE}

To solve the problem, a new block-matching algorithm using adjustable search patterns is proposed. Adjustable search patterns mainly contain three techniques:

(1) Adaptive threshold values for early termination of blockmatching. To set different threshold values according to the motion vectors of their adjacent blocks to determine the relatively stationary blocks.

(2) Block classification. Divide the non-static macro blocks into three categories - smooth, medium and violent and use different estimation methods according to the motion intensity.

(3) Local optimum sticking avoidance using ABCA. NTSS is chosen for the smooth blocks with small motion vectors. Medium blocks with relatively large motion are processed by DS. For the violent blocks, ABCA is applied to avoid getting trapped in local optimum.

(4) The aim is to find the corresponding block among the candidate blocks within the search window in the reference frame for the current block in the current frame and acquire its motion vector. During the process, both estimation accuracy and computational complexity are important factors to consider about. In this research, we introduce an adaptive threshold value for early termination of block-matching technique, a local optimum sticking avoidance using technique, and make use of the temporal-spatial correlation of the motion vectors to classify macro blocks to finally propose a novel adaptive block-matching algorithm using modified adjustable search patterns which performs very well both in estimation accuracy and computational complexity. In the following, the three main techniques of the proposed algorithm are discussed. 
(5) In this section, we'll firstly introduce a basic early termination of block-matching technique with fixed threshold value. Then an adaptive threshold values for early termination of block-matching technique is proposed. For videos with flat motion, most of the blocks are stationary. It is a big waste of time to run blockmatching algorithm for these blocks. Therefore, early termination of block-matching technique is proposed. It decides whether to abandon the search process of the current block in terms of a certain criterion such as:

$S A D_{0}=\sum_{x=1}^{N} \sum_{y=1}^{N}\left|f_{k}(x, y)-f_{k-1}(x, y)\right|$

(6) If $S A D \leq S$ the current block is considered as stationary, the algorithm for the current block is interrupted to save computational complexity. According to, experimental statistics have shown that more than ninety-eight percent of the stationary block's $S A D$ value is less than 512 .

In any case, the current early end of square coordinating system needs adaptability in deciding stationary squares for it embraces just single settled limit esteem. It is clear that the nature of the reproduced picture is to a great extent chosen by the estimation precision of the generally huge movement obstructs in that outline. A forfeit of the scan procedure for the generally little movement squares can bring huge conservation of the time cost with only an unimportant loss of precision. Moreover, truth be told, stationary or not is outwardly relative. Some insignificant movement encompassed by substantial movement or the generally little movement following the vast movement is difficult to distinguish by eyes. Thusly, the pursuit productivity will be enhanced a great deal, if the limit esteems are adaptively set by the relative movement power of the related blocks.

\section{RESULTS}

The threshold values can be flexibly set according to the format of the video sequence. For example, for the QCIF sequences, most of the blocks are small motion blocks, the threshold value of other threshold point should be relatively larger in case of ignoring too many small motion vectors to achieve satisfactory estimation accuracy. During the whole searching, most of the time is spent on the process of calculating the cost function value between the reference block and the candidate blocks, while the other processes just account for a trivial part which is also partly influenced by the optimization degree of the code

\section{CONCLUSION}

The focus of this research is on the block matching algorithms which are used for measuring the computational complexity of the motion sequences. More precisely, the aim is to find the optimized method for improving the computational efficiency of the algorithms for motion estimation. The literature focuses on core technology and the framework of H.264/AVC and then compares the pros and cons of some classic motion estimation algorithms.

The results of the implemented technique shows that the computational complexity is reduced for motion estimation while keeping the same quality of the video as the pixels are not distorted or disturbed while the searching of blocks. The CIF and QCIF video samples are tested for the implemented technique. In normal scenarios it takes around ninety percent for the computations of motion estimation. Noise is introduced in motion sequences through bidirectional motion estimation methods.

In order to speed up the searching process in motion sequences, a method is proposed which focuses on bidirectional search. The proposed algorithm simulation results shows minimum error and less number of search points when compared to the existing algorithms.

\section{REFERENCES}

[1] Verma, V., \& Mishra, R. (2013). Various Fast Block Matching Algorithm for Video Shot Boundary Detection. IOSR Journal of Electrical and Electronics Engineering (IOSR-JEEE), vol.4, no. 1, pp. 11-17.

[2] Chandra Sekhar, C.H., \& Ratnam, J.V.K. (2012) "Comparison of Fast Block Matching Algorithms for Motion Estimation", International Journal of Electronics and Computer Science Engineering (IJCSE), vol.1, no. 3, pp. 1609-1618.

[3] Immanuel, S., Pandian, A., \& George, B.A. (2011) "A Study on Block Matching Algorithms for Motion Estimation”, International Journal on Computer Science and Engineering (IJCSE), vol. 3, no. 1, pp. 34-44.

[4] Stiller, C., \& Konrad, J. (1999) "Estimating motion in image sequences", IEEE Signal Processing Magazine, vol. 16, no. 4, pp. 70-91.

[5] Arvanitidou, M. G., Tok, M., Glantz, A., Krutz, A., \& Sikora, T. (2013) "Motion-based object segmentation using hysteresis and bidirectional inter-frame change detection in sequences with moving camera", Signal Processing: Image Communication, vol. 28, no. 10, pp. 1420-1434.

[6] Pradhan, C., \& Adak, D. (2012). "Survey on block matching algorithms for motion estimation", International Journal of Computer Applications, vol. 46, no. 16, pp. 6-10.

[7] Bouthemy, P. (2004). "2D motion description and contextual motion analysis: Issues and new models", International Workshop on Spatial Coherence for Visual Motion Analysis, Springer, Berlin, Heidelberg. pp. 1-15.

[8] Rodriguez, A., Fernandez-Lozano, C., Dorado, J., \& Rabuñal, J. R. (2014) "Two-Dimensional Gel Electrophoresis Image Registration Using BlockMatching Techniques and Deformation Models." Analytical Biochemistry, 454.1, pp. 53-59.

[9] Ng, K. H., Po, L. M., Wong, K. M., Ting, C. W., \& Cheung, K. W. (2009) "A search patterns switching algorithm for block motion estimation", IEEE Transactions on Circuits and Systems for Video Technology, vol. 19, no. 5, pp. 753-759.

[10] Ng, K. H., Po, L. M., Wong, K. M., Ting, C. W., \& Cheung, K. W. (2009) "Multiple block-size search algorithm for fast block motion estimation", IEEE 7th International Conference on Information, Communications and Signal Processing (ICICS), pp. 1-4.

[11] Jagiwala, D. D., \& Shah, M. S. (2012) "Analysis of block matching algorithms for motion estimation in $\mathrm{H}$. 264 video CODEC", Analysis, vol. 2, no. 6, pp. 13961401.

[12] Metkar, S., \& Talbar, S. (2013) "Performance evaluation of block matching algorithms for video coding", Motion Estimation Techniques for Digital Video Coding, 
Springer, India, pp. 13-31,

[13] Rodriguez, A., Rabuñal, J. R., Pérez, J. L., \& Martínez- Abella, F. (2012) "Optical analysis of strength tests based on block-matching techniques", Computer-Aided Civil and Infrastructure Engineering, vol. 27, no. 8, pp. 573-593.

[14] Tourapis, A. M., Wu, F., \& Li, S. (2005) "Direct mode coding for bipredictive slices in the H. 264 standard" IEEE Transactions on circuits and systems for video technology, vol. 15, no. 1, pp. 119-126.

[15] Freire, A., Seoane, J. A., Rodríguez, Á., Ruiz-Romero, C., López-Campos, G., \& Dorado, J. (2010) "A Blockmatching based technique for the analysis of $2 \mathrm{D}$ gel images", MedInfo, pp. 1282-1286.

[16] Mishra, S. S., Pradhan, C., \& Singh, A. (2014) "Comparative study of motion estimation techniques in video", Int. J. Comput. Sci. Inf. Technol, vol. 5, no. 3, pp. 2982-2989.

[17] Cheng, S. C., \& Hang, H. M. (1997) "A comparison of block-matching algorithms mapped to systolic-array implementation", IEEE Transactions on Circuits and Systems for Video Technology, vol. 7, no. 5, pp. 741-757.

[18] Seferidis, V. E., \& Ghanbari, M. (1993) "General approach to block-matching motion estimation", Optical Engineering, vol. 32, no. 7, pp. 1464-1475.

[19] Nie, Y., \& Ma, K. K. (2002) "Adaptive rood pattern search for fast block-matching motion estimation", IEEE Transactions on Image processing, vol. 11, no.12, pp.
$1442-1449$

[20] Manjunatha, D. V., \& Sainarayanan, D. (2011) "Comparison and implementation of fast block matching motion estimation algorithms for video compression", Int. J. Eng. Sci. Technol.(IJEST), vol. 3, no. 10 , pp. $7608-7613$

[21] Rajamanickam, V., \& Marikkannan, S. (2016) "Efficient Block-Based Motion Estimation Architecture Using Particle Swarm Optimization”, Int. Arab J. Inf. Technol., vol. 13, no.6A, pp. 732-739.

[22] Cai, J., \& Pan, W. D. (2010) "Fast exhaustive-search motion estimation based on accelerated multilevel successive elimination algorithm with multiple passes", IEEE International Conference on Acoustics, Speech and Signal Processing, pp. 1190-1193.

[23] Korat, P. N., \& Bhojani, D. R. (2014) "A new hybrid block-based motion estimation algorithm for video compression", Int. J. Adv. Res. Electr., Electron. Instrum. Engg, pp. 9586-9596.

[24] Tok, M., Eiselein, V., \& Sikora, T. (2015) "Motion modeling for motion vector coding in HEVC", IEEE Picture Coding Symposium (PCS), pp. 154-158.

[25] Po, L. M., Ng, K. H., Cheung, K. W., Wong, K. M., Uddin, Y. M. S., \& Ting, C. W. (2009) "Novel directional gradient descent searches for fast block motion estimation", IEEE Transactions on Circuits and Systems for Video Technology, vol. 19, no. 8, pp. 11891195. 